Hias Julie (Orcid ID: 0000-0002-7269-2515)

Mian Paola (Orcid ID: 0000-0002-3551-1201)

\title{
Pharmacokinetics of two oral paracetamol formulations in hospitalized octogenarians
}

\section{Short running title: PK of paracetamol in octogenarians}

Authors: Julie Hias ${ }^{1}$, Lorenz Van der Linden ${ }^{1,2}$, Karolien Walgraeve ${ }^{1}$, Matthias Gijsen ${ }^{1,2}$, Paola Mian ${ }^{3}$, Birgit C P Koch ${ }^{4}$, Karel Allegaert ${ }^{2,4,5}$, Pieter Annaert ${ }^{6}$, Jos Tournoy $^{7,8}$, Isabel Spriet ${ }^{1,2}$

\section{Affiliations:}

1. Pharmacy Department, University Hospitals Leuven, Leuven, Belgium

2. Department of Pharmaceutical and Pharmacological Sciences, KU Leuven University of Leuven, Leuven, Belgium

3. Department of Clinical Pharmacy, Medisch Spectrum Twente, Enschede, The Netherlands

4. Department of Hospital pharmacy, Erasmus MC University Medical Center, Rotterdam, The Netherlands

5. Department of Development and Regeneration, KU Leuven - University of Leuven, Leuven, Belgium

6. Drug Delivery and Disposition, Department of Pharmaceutical and Pharmacological Sciences, KU Leuven - University of Leuven, Leuven, Belgium

7. Department of Geriatric Medicine, University Hospitals Leuven, Leuven, Belgium

8. Department of Public Health and Primary care KU Leuven - University of Leuven, Leuven, Belgium

\section{Corresponding author details:}

Julie Hias

E-mail: julie.1.hias@uzleuven.be

Address: Department of Hospital Pharmacy, Herestraat 49, 3000 Leuven, Belgium

Phone: +3216343080; Fax: +3216343085

The authors confirm that the Principal investigator for this paper is Jos Tournoy and that he had direct clinical responsibility for patients.

This article has been accepted for publication and undergone full peer review but has not been through the copyediting, typesetting, pagination and proofreading process which may lead to differences between this version and the Version of Record. Please cite this article as doi: $10.1111 / \mathrm{bcp} .15049$ 


\section{Keywords}

Pharmacokinetics, paracetamol, older inpatients

\section{Word counts}

Abstract: 250

Main text: 3777

Number of tables: 5

Number of figures: 2

What is already known about this study?

- Oral paracetamol is the agent of choice for managing pain in older adults and dosing recommendations are extrapolated from data in younger adults.

- Ageing is associated with altered pharmacokinetics of intravenous paracetamol.

- Pharmacokinetics of oral paracetamol in very old frail patients are insufficiently characterized.

What this study adds

- Large interindividual variability in pharmacokinetics were observed.

- No difference in absorption was observed between the granulate and tablet formulation.

- Female sex and higher total serum bilirubin concentration were associated with an increased paracetamol exposure in an exploratory analysis. 


\section{ABSTRACT}

AIM

It is currently unclear how paracetamol (acetaminophen) should be dosed in order to increase its efficacy while warranting safety in very old adults. The objective was to evaluate the pharmacokinetics of two oral paracetamol formulations and its metabolites in hospitalized octogenarians.

\section{METHODS}

Geriatric inpatients aged 80 years and older received a 1000mg paracetamol tablet or granulate at 8AM, 2PM and 8PM. After at least four consecutive gifts, plasma samples were collected around the $8 \mathrm{AM}$ dose [trough, $+0.5,+1,+2,+4,+5$ and +6 hours]. Plasma concentrations of paracetamol and its metabolites were determined and individual pharmacokinetic parameters were derived. The Edmonton Frail Scale was used to assess frailty. An analgesic plasma target was defined as an average plasma concentration (Cavg) of $10 \mathrm{mg} / \mathrm{L}$.

\section{RESULTS}

The mean $( \pm S D)$ age was $86.78( \pm 4.20)$ years. The majority $(N=26 / 36,72 \%)$ received the tablet, $10(28 \%)$ the granulate. Thirty patients (85\%) were classified with moderate to severe frailty. Seven (21\%) patients had a Cavg above $10 \mathrm{mg} / \mathrm{L}$. The median [IQR] time to reach the peak concentration $\left(T_{\max }\right)$ was 50.5 [31.50-92.50] and 42.50 [33.75106.75] min for the tablet and granulate, respectively. The coefficient of variation was $95 \%$ for $T_{\max }$ and $30 \%$ for Cavg of paracetamol. A correlation of Cavg of paracetamol was observed with female sex and total serum bilirubin.

\section{CONCLUSION}

Large interindividual differences were found for pharmacokinetic parameters of oral paracetamol in frail inpatients after multiple dosing. Female sex and higher total serum bilirubin concentrations were associated with paracetamol exposure. No significant differences were observed between the tablet and granulate. 


\section{BACKGROUND}

The prevalence of chronic pain is high in very old adults and inadequate pain control remains a significant issue in this population [1-4]. Multiple causes can be identified. First, pain in very old adults is often underreported and might be related to cognitive impairment, which can lead to difficulties in pain assessment. Secondly, a lacking knowledge of healthcare professionals on how to provide appropriate pain treatment might further impact pain control. Thirdly fear of potential adverse drug events might add to the overall issues of undertreatment in very old adults [5]. Most mild-tomoderate pain syndromes in very old adults are initially managed with paracetamol (acetaminophen), which is in accordance with the World Health Organization (WHO) analgesic ladder. The WHO recommends to use non-opioid minor analgesics such as paracetamol as first-line treatment of pain. Addition of a weak opioid, such as tramadol or codeine, is suggested if pain is not properly controlled. Next, weak opioids can be substituted by more potent opioid, such as oxycodone or morphine [6]. As nonsteroidal anti-inflammatory drugs (NSAIDs) are associated with an increased risk for adverse drug events, these are not considered first-line agents in this patient population [7].

Dosing recommendations for paracetamol are available, yet none with a specific focus on patients aged 80 years and older [2]. As a result, paracetamol is mainly dosed according to clinical experience, expert opinion or based on pharmacokinetic data extrapolated from studies in younger adults [2]. Previous research by Mian et al showed that ageing coincided with a decreased volume of distribution $(\mathrm{Vd})$ and total paracetamol clearance from plasma $(\mathrm{CL})$. Yet, a clear impact of age on paracetamol absorption was not observed [2]. More data are urgently needed to better understand how physiological changes during ageing impact the pharmacokinetics as well as the analgesic effects of oral paracetamol, in particular in frail older inpatients [2].

Furthermore, it has been suggested to aim for a target plasma concentration of 10 $\mathrm{mg} / \mathrm{L}$ to manage pain adequately. Similarly, this target has never been studied or validated in adults aged 80 years and older [2, 8]. A population pharmacokinetic study has proposed practical dosing guidelines for older adults receiving intravenous paracetamol. In this single-dose pharmacokinetic study, investigators mostly enrolled fit and robust older adults with a median age of 77.3 years following orthopedic surgery [9]. In summary, there is a need for data on paracetamol pharmacokinetics in frail adults aged 80 years and older receiving oral paracetamol during multiple dosing. These data might then be used for pharmacodynamics studies and subsequently to inform an evidence-based dosing regimen of oral paracetamol in older adults.

The aims of this study were hence to determine exposure and pharmacokinetics of oral paracetamol and its metabolites in geriatric inpatients aged 80 years and older during multiple dosing and to investigate whether a tablet vs. granulate formulation may affect absorption and subsequent exposure. 


\section{METHODS}

\section{Design and setting}

An observational monocentric pharmacokinetic study was conducted. The study was organized at the 80-bed acute geriatric department of the 1950-bed University Hospitals Leuven in Belgium. The study was approved by the local Ethics Committee (S58396) and was registered at ClinicalTrials.gov (Identifier: NCT03617471).

\section{Study participants}

Patients were eligible for study participation if the following criteria were met: written informed consent provided by the patients or their relatives in case of inability to provide the consent themselves; a minimum age of 80 years; admission to the acute geriatric ward; oral intake of paracetamol, treatment with 1000 milligrams $(\mathrm{mg})$ in tablet or granulate formulation at the discretion of the treating physician, three times daily (8AM, 2PM, 8PM); at least four consecutive gifts of paracetamol administered before sampling. Patients were excluded in case of a do-not-resuscitate code corresponding to active withdrawal of care (= "end of life care").

\section{Pharmacokinetic sampling}

Blood sampling for paracetamol and its metabolites was performed during one dosing interval of oral paracetamol starting at 8AM after at least four confirmed consecutive intakes. During a first inclusion period (November 2015 - January 2016) only patients taking the tablet formulation (Dafalgan Forte $\AA$, tablets, Bristol-Myers Squibb Belgium N.V., Brussels, $1000 \mathrm{mg}$ ) were included. During a second inclusion period (July November 2016) a new formulation (Dafalgan Instant forte $\AA$, granulate, Bristol-Myers Squibb Belgium N.V., Brussels, 1000mg) was introduced; patients taking this formulation were included as well.

In the first study period, six blood samples were collected via a peripheral venous catheter: within 15 minutes before intake of the 8AM dose (trough level, T0, i.e., 12 hours after intake last paracetamol) and at +0.5 (T0.5), +1 (T1) +2 (T2), +4 (T4) and +6 hours (T6, trough level before intake of second paracetamol at 2PM) after oral administration of paracetamol. In the second study period (July - November 2016) a seventh sample drawn at +5hours (T5) after paracetamol intake was introduced allowing for a better estimation of the elimination phase. Samples were centrifuged at $3000 \mathrm{rpm}$ for 15 minutes immediately after collection. Plasma was stored at $-20^{\circ} \mathrm{C}$ until bio-analysis.

\section{Bio-analysis}

Analysis of paracetamol concentrations and its major metabolites paracetamolglucuronide, paracetamol-sulphate, paracetamol-mercapturic acid and paracetamolcysteine was performed at the Hospital Pharmacy Laboratory of the Erasmus MC Pharmacy department using an Ultra Performance Liquid Chromatography - tandem mass spectrometry (UPLC-MS/MS) method. The equipment used was a Dionex Ultimate UPLC system consisting of an Ultimate 3000 RS UPLC pump, an Ultimate 3000 RS autosampler, and an Ultimate 3000 RS Column Compartment. The UPLC was connected to a triple quadrupole Thermo TSQ Vantage MS with HESI probe 
(Thermo Scientific, Waltham, MA). The software programs Chromeleon (version 6.8; Dionex, Thermo Scientific), Xcalibur (version 2.1; Thermo Scientific), and LCquan (version 2.6; Thermo Scientific) were used to control the system and analyze the data. The assays were linear from 0.020 to $25.0 \mathrm{mg} / \mathrm{L}, 0.047$ to $47.0 \mathrm{mg} / \mathrm{L}$, and 0.043 to $43.0 \mathrm{mg} / \mathrm{L}$ for paracetamol, paracetamol-glucuronide, and paracetamol-sulfate, respectively, and from 0.020 to $10.0 \mathrm{mg} / \mathrm{L}$, and 0.010 to $15 \mathrm{mg} / \mathrm{L}$ for paracetamolcysteine and paracetamol-mercapturic acid. The lower limit of the ranges represents the lower limits of quantification (LLOQ). Intra- and interassay accuracies ranged from $93.6 \%$ to $130.9 \%$. Intra- and interassay imprecision did not exceed $15 \%$. More details are described in an earlier validation article. The method was validated according to Food and Drug Administration guidelines [10].

\section{Patient variables}

The following patient variables were retrieved from the electronic patient health record: age, sex, body length, body weight and Body Mass Index (BMI). Biochemical parameters from the last available blood sample prior to paracetamol sampling were acquired as follows: serum albumin concentrations, estimated glomerular filtration rate (eGFR) according to the CKD-EPI (chronic kidney disease-epidemiology collaboration) formula and estimated creatinine clearance according to the Cockcroft and Gault ( $\mathrm{CrClcG}$ ) formula, serum creatinine $(\mathrm{SCr})$ total serum bilirubin, direct bilirubin, aspartate aminotransferase (AST), alanine aminotransferase (ALT), gammaglutamyltransferase (GGT) and alkaline phosphatase concentrations. The values were documented as reported in the electronic health record. Comorbidities such as diabetes mellitus, chronic obstructive pulmonary disease (COPD), heart failure, chronic kidney disease, dementia, chronic pain, liver disease, anorexia and Parkinson's disease were registered as well. Additional variables were collected as follows: the indication for paracetamol administration (pain, fever or both), the number of concomitant drugs, the use of other analgesics, the pain score determined by the numeric rating scale (NRS) or the Pain Assessment IN Advanced Dementia (PAINAD) tool at each sample moment (T0-T6), the Mini Mental State Examination (MMSE) score and frailty status determined by the Edmonton Frail Scale (EFS) [11, 12].

\section{Pharmacokinetic parameters}

Individual pharmacokinetic parameters were determined by non-compartmental analysis using Microsoft Excel Windows 2016 (16.0.6366.2062 21 January 2016). The terminal elimination rate $\left(\mathrm{k}_{\mathrm{e}}\right)$ constant was estimated by linear regression of the natural logarithms of mean plasma concentrations versus time. The area under the plasma concentration-time curve $\left(\mathrm{AUC}_{0-6}\right)$ was calculated by the linear up/log down trapezoidal method. The half-life $\left(t^{1} / 2\right)$ was calculated as $\ln 2 / k_{e}$. Oral plasma clearance $(\mathrm{CL} / \mathrm{F})$ was calculated as dose/AUC $\mathrm{C}_{0-6}$. Volume of distribution $(\mathrm{Vd})$ was calculated as dose/(ke.AUC 0 -6). Maximum plasma concentration $\left(\mathrm{C}_{\max }\right)$ and time to reach $\mathrm{C}_{\max }\left(\mathrm{T}_{\max }\right)$ of paracetamol were observed directly from the data. Average plasma concentration (Cavg) was calculated as $A \cup C_{0-6 / 6}$. The AUC of the metabolites (in $\mathrm{mg}^{*} \mathrm{~h} / \mathrm{L}$ and $\mathrm{mmol}{ }^{*} \mathrm{~h} / \mathrm{L}$ ) and the metabolite-to-parent drug AUC ratios (AUCmo-6/AUCp 0 -6) were calculated (in $\mathrm{mg}$ and $\mathrm{mmol}$ ) as well. 
Pharmacokinetic parameters were compared to previously reported study findings, where paracetamol, in a dose of $1000 \mathrm{mg}$ or $14 \mathrm{mg} / \mathrm{kg}$, administered intravenously or orally, was examined in patients with a mean age of 75 years or older and to their comparison groups of younger adults.

Target attainment was determined as a Cavg above the analgesic target of $10 \mathrm{mg} / \mathrm{L}$ [8].

\section{Statistical analysis}

Normality of the continuous variables was evaluated by visual inspection of the histograms and QQ plots. Variables were reported as mean ( \pm standard deviation (SD)) or median [interquartile range, IQR $=\mathrm{Q} 1-\mathrm{Q} 3$ ], as appropriate. Proportions and counts were represented as $n(\%)$. The coefficient of variation (CV) was reported for $T_{\max }$, $\mathrm{C}_{\max }$ and Cavg for paracetamol. CV is defined as the ratio of the SD to the mean and expressed as a percentage. Differences in pharmacokinetic parameters between the different formulations of paracetamol were determined using the Mann Whitney U-test.

Univariate linear regression analyses with Cavg of paracetamol and each of the metabolites as separate outcome variables were performed to investigate the relationship with body weight, body mass index, total serum bilirubin concentrations, sex, CrClcG, GGT and serum albumin concentrations. The variables showing a statistically significant relationship were included along with the type of formulation in the multivariable regression analysis. Statistical significance was established as $p<0.05$, with all tests being two-tailed.

Data were analyzed using the Statistical Package for the Social Sciences (IBM Corp. Released 2020. IBM SPSS Statistics for Windows, Version 27.0. Armonk, NY: IBM Corp.) and $\mathrm{R}$ software (R version 3.5.1; The R Foundation for Statistical Computing, Vienna, Austria).

\section{RESULTS}

A total of 36 patients were included for pharmacokinetic analysis. The patient selection process has been summarized in Figure 1.

The patients' mean age was $86.78( \pm 4.20)$ years. Paracetamol was prescribed for pain in $89 \%$, fever in $8 \%$ and for both indications in $3 \%$ in patients. Almost half of the patients (49\%) received other analgesics during paracetamol treatment and in 33\% chronic pain was mentioned in their medical history. Other demographic characteristics, are shown in Table 1.

A decrease in pain score determined by the numeric rating scale (NRS) between the moment before the intake of paracetamol and one hour after the intake was observed in $40 \%(n=10 / 25)$ of patients, the score remained the same in $36 \%(n=6 / 25)$ of patients and increased in $24 \%(n=9 / 25)$. The median paracetamol plasma concentration-time curve of all patients is displayed in Figure 2.

A minority of patients, i.e. $7 / 33$ (21\%), had a Cavg exceeding the predefined analgesic target of $10 \mathrm{mg} / \mathrm{L}$. Pharmacokinetic parameters have been summarized in Table 2, 
which also contains data from previously published studies on the use of paracetamol in older adults aged 75 years or older and their comparator groups of fit younger patients. CVs were $95 \%$ for $\mathrm{T}_{\max }, 35 \%$ for $\mathrm{C}_{\max }$ and $30 \%$ for Cavg of paracetamol.

Ten patients took the granulate formulation. Pharmacokinetic parameters did not differ significantly between patients receiving a tablet or a granulate formulation. Details are shown in Table 3.

The AUCs for the main paracetamol metabolites and the metabolite/paracetamol ratio have been summarized in Table 4. Data from Liukas et al. are shown in the table as well. The $A_{U} C_{0-6}$ expressed in mmol*h/L were 0.3 [0.02-0.04], 0.59 [0.48-0.76], 0.006 [0.004-0.008] and 0.28 [0.20-0.37] mmol*h/L for the cysteine, glucuronide, mercapturic acid and sulphate conjugates, respectively. The metabolic ratios expressed in $\mathrm{mmol}$ were 0.07 [0.05-0.15], 1.73 [1.47-2.94], 0.019 [0.012-0.024] and 0.79 [0.64-1.52] for the cysteine, glucuronide, mercapturic acid and sulphate conjugates, respectively.

Total serum bilirubin concentrations and sex were included in the multivariable regression analysis along with the type of formulation. A higher total serum bilirubin concentration and female sex were significantly associated with an increased Cavg.

For Cavg of the paracetamol metabolites GGT, CrClcG and the type of formulation were included in the separate multivariable regression analyses for each metabolite. CrClcG showed an inverse association with Cavg for each of the metabolites in the multivariable model. For the cysteine-metabolite, also increased GGT was associated with an increased Cavg. The results of the multivariable regression analyses are shown in Table 5.

\section{DISCUSSION}

In this study we determined pharmacokinetics and exposure of oral paracetamol and its metabolites in frail geriatric inpatients aged 80 years or older during multiple dosing. To the best of our knowledge, our study is presently the largest dataset in this complex patient population (Table 2). Importantly, we observed a large interindividual variability, as demonstrated by the wide IQR for all pharmacokinetic parameters and relatively high CVs for Tmax, Cmax and Cavg. In the majority of patients (26/33, 79\%) the concentration at steady state was below the predefined analgesic target of $10 \mathrm{mg} / \mathrm{L}$ as shown by a median [IQR] Cavg of 8,00 [6.16-9.34] mg/L resulting in an overall low exposure. No significant differences were seen in exposure following the tablet versus granulate formulations. The pain levels of the majority of patients remained the same or decreased one hour after paracetamol intake.

We believe our study results are valid, yet some limitations have to be taken into consideration. First, only patients aged 80 years or older were included, hence no direct comparison with fit younger adults (i.e. younger than 80 years) was possible. However, we compared our results to already published data. Second, all included published data extrapolated their AUC to infinity which made the comparison with our data challenging. Extrapolation to infinity is usually done in studies reporting single dose pharmacokinetics, however, in our study sampling was performed when the 
patient had taken at least four consecutive doses of paracetamol. This pragmatic study design allows for better insights into paracetamol pharmacokinetics during multiple dosing, which is closer to the daily clinical practice. Third, paracetamol was prescribed at $8 \mathrm{AM}, 2 \mathrm{PM}$ and $8 \mathrm{PM}$. The intervals between consecutive doses were hence different: the first trough level taken at 8AM was 12 hours after the last paracetamol intake and the last trough level taken at 2PM was 6 hours after the morning intake of paracetamol. However, this represents daily practice in most hospital settings, since oral therapy is commonly administered throughout the day, thus further adding to the external validity of our data. Fourth, mean and SD were often used to describe data in the literature. In contrast, we reported median and IQR since none of our evaluated pharmacokinetic parameters were distributed normally. We believe median and IQR better represent the highly variable pharmacokinetics of paracetamol in our cohort. Fifth, the analgesic target of $10 \mathrm{mg} / \mathrm{L}$ is debatable. It is based on studies predominantly performed in pediatric patients after surgery and was not validated for geriatric patients. Based on the available body of evidence, Gibb et al concluded however that a target of $10 \mathrm{mg} / \mathrm{L}$ for pain control could be used for adults. Since this cut-off it is the only one reported to be associated with adequate analgesia, this was applied to define target attainment [8]. No hard conclusions should be drawn however based on target attainment as its clinical relevance in our patient population remains uncertain. A formal exposure (e.g. Cavg) vs. response (pain assessment, using NRS or PAINAD) relationship analysis should be carried out in older adults with a geriatric profile in order to identify validated targets.

The following strengths add to the value of our data. First, the sample size of our study population $(n=36)$ exceeds that of other pharmacokinetic studies with paracetamol, which ranged from 6 to 26 patients [13-17]. Second, two different oral formulations of the study drug were compared. This strengthens the belief that variability is rather linked to the oral route of administration than to the formulation. Third, the major metabolites of paracetamol, i.e. the sulphate, glucuronide, mercapturic acid and cysteine conjugates, were analyzed as well, allowing for more detailed insights regarding the elimination of paracetamol in frail patients aged 80 years and older.

Table 2 shows a comparison with published data of paracetamol pharmacokinetic parameters. Compared to younger adults, the AUC was similar in our frail elderly population [15]. The $\mathrm{CL} / \mathrm{F}$ found in this study was lower than those reported by Triggs et al and Miners et al [14, 16]. Mian et al. also concluded in their review that CL/F decreased with age and frailty [2]. The $\mathrm{Vd}$ was slightly lower than the values as reported by Liukas et al and Triggs et al $[14,15]$. A decrease in Vd with increasing age and frailty was also described by Mian et al [2].

The population investigated by Ellmers et al was comparable to ours. They enrolled frail older patients with an average age of 83.5 years taking oral paracetamol 1000 mg. Compared to their results, our average AUC was reduced, CL/F was increased and $t_{1 / 2}$ was prolonged [13]. Furthermore, our average AUC was reduced and CL/F increased in comparison to other previously published findings in fit patients aged 75 years or older $[13,15,17]$. T max was prolonged compared to most other investigations on oral paracetamol in fit older patients aged 75 years and older [14 1988, Comparison of paracetamol metabolism in young adult and elderly males, 16]. C $\max$ was lower

This article is protected by copyright. All rights reserved. 
compared to the results of the fit older adults included by Bannwarth et al and comparable to the results found by Miners et al in the same population [16, 17 1988, Comparison of paracetamol metabolism in young adult and elderly males]. Vd and $\mathrm{t}_{1 / 2}$ were comparable to other trial results in fit older adults [13-17].

Since $\mathrm{CL} / \mathrm{F}$ is directly linked to $\mathrm{AUC}(\mathrm{CL}=\mathrm{D} / \mathrm{AUC})$, we hypothesize that the lower paracetamol exposure in our study population might be explained by reduced absorption, reflected by a delayed $T_{\max }$ and lower $\mathrm{C}_{\max }$. In the granulate group, a shorter average $T_{\max }$ and higher average Cavg, hinting toward increased absorption, were observed. However, no statistically significant differences between the tablet and granulate groups were observed for any of the pharmacokinetic parameters. Patients who received the granulate formulation had an average Cavg of 9.27 [4.94-11.03] $\mathrm{mg} / \mathrm{l}$, which is closer to the target of $10 \mathrm{mg} / \mathrm{l}$. Results were nonetheless highly variable as demonstrated by the wide IQR and relatively high CVs. Importantly, although explorative, these results suggest that facilitating the dissolution of paracetamol, by using a granulate formulation, did not affect absorption of paracetamol in a meaningful manner. Other factors such as the rate of gastric emptying could perhaps explain the delayed and reduced absorption and the subsequent lower exposure in our study sample [18]. The lack of a statistically significant difference between the two formulation might be explained by the small sample size and deserves further investigation. More research is necessary for factors influencing the absorption process of paracetamol and other drugs in frail older patients.

Female sex and a higher total serum bilirubin concentration were statistically associated with an increase in Cavg of paracetamol. Liver toxicity is a known adverse drug reaction of paracetamol overdose, but it is also described with therapeutic doses [19]. A higher serum bilirubin concentration could be a sign of liver toxicity and could be a result of an increased paracetamol exposure after multiple dosing. However, only two patients had a total bilirubin concentration above the upper limit of normal of 1.18 $\mathrm{mg} / \mathrm{dl}$. Female patients had a higher exposure which was also observed by Liukas et al [15]. This is an exploratory analysis for covariates influencing paracetamol exposure. Therefore, these results need to be confirmed in a larger patient group to allow for a robust multivariable analysis of the correlation between patient characteristics and paracetamol exposure.

Pain scores were determined, yet interpretation was difficult and not linked to any of the pharmacokinetic parameters of paracetamol or its metabolites. Firstly, while both the Numerical Rating Scale or Pain Assessment IN Advanced Dementia tools were are regularly used on our geriatric wards to determine the pain scores only the NRS score results were registered for all included patients. Knowing that $47 \%$ of patients included in our study were diagnosed with dementia, using the NRS was not considered appropriate. Secondly, it was not clear for which type of pain paracetamol was prescribed. In very old patients paracetamol is often prescribed instead of nonsteroidal anti-inflammatory drugs (NSAIDs) for musculoskeletal pain, owing to the negative benefit/risk ratio of the latter, even though they have shown to be more effective analgesics [20]. Thirdly, the majority ( $n=17,49 \%)$ of patients also received other analgesics, such as NSAIDs (6\%) and mild (53\%) or strong (47\%) opioids. This 
complicates the evaluation of the correlation between pharmacokinetic parameters and pharmacodynamic effects such as analgesic effects for paracetamol.

The major metabolites of paracetamol are the sulphate and glucuronide conjugates, but a minor fraction is converted to a highly reactive metabolite, $\mathrm{N}$-acetyl-pbenzoquinone-imine (NAPQI). Normally, this metabolite is rapidly inactivated by conjugation with reduced glutathione and is excreted in urine as cysteine and mercapturic acid conjugates [21]. In our population, the metabolite/paracetamol ratio was the highest for the glucuronide conjugate, followed by the sulphate conjugate. The ratios were similar compared to the ratios found by Liukas et al in their group aged 80 years and older. Yet, we should refrain from overinterpreting the comparison to the Liukas data. Our study was performed in the setting of multiple dosing and hence steady state was assumed to be reached [15]. Very low ratios for the mercapturic acid and cysteine conjugates were found in our cohort. The analytic method was not able to discern NAPQI concentrations and toxic effects were not evaluated. An inverse correlation was found between the $\mathrm{CrCl}_{C G}$ and the AUCs of all conjugates. This association is in line with previous results by Liukas et al for the sulphate and glucuronide conjugates [15]. In sum, decreased renal function might be associated with an accumulation of paracetamol metabolites. Further research should determine the clinical significance of this accumulation.

Due to the large interindividual differences, a population pharmacokinetic model is warranted in order to explain the variability and to determine an optimal dosing regimen for this specific population of octogenarians. Safety studies concerning hepatotoxicity are also needed to optimize the paracetamol dosage guidelines for frail older patients [22].

\section{CONCLUSION}

Exposure to paracetamol and its metabolites, after administration of multiple oral doses of the tablet vs. granulate formulation, was documented for the first time in a large cohort of frail inpatients aged 80 years and older. A large interindividual variability in exposure and pharmacokinetic parameters was found. The granulate formulation did not lead to significantly higher exposure; pharmacokinetic parameters were neither significantly different when comparing both formulations. Female sex and higher total serum bilirubin concentrations were found to be associated with increased paracetamol exposure. Ideally, a well-designed pharmacokinetic-pharmacodynamic modeling and simulation analysis, in which exposure is analyzed in relation to pain assessment scores, is needed to understand the relation between exposure and response and the need for dosing optimization.

\section{Acknowledgements:}

The authors wish to thank all students (Ebe Sacré, Hanne Vandeput, Ineke Van der Biest, Alicia Declercq, Hanne Reynaert and Simon Volcke) for patient inclusion and data collection. We also wish to thank Azuka Chude, Alicia Declercq, Hanne Reynaert and Simon Volcke for collecting the blood samples.

Authorship:

This article is protected by copyright. All rights reserved. 
$\mathrm{JH}, \mathrm{LVdL}, \mathrm{IS}, \mathrm{JT}$ and $\mathrm{KW}$ have made substantial contributions to the concept and design, acquisition of data, analysis of data, interpretation of data and were involved in drafting the manuscript. KA, PM, PA and BCPK were involved in revising the manuscript critically for important intellectual content. MG was involved in revising the manuscript by running the multivariable analysis. All authors gave final approval of the version to be published.

\section{Conflict of interest:}

The authors declare that they have no conflict of interest

\section{Funding:}

No specific funding was received to perform this study

\section{Data availability statement:}

The data that support the findings of this study are available from the corresponding author upon reasonable request.

\section{Nomenclature of Targets and Ligands:}

Key protein targets and ligands in this article are hyperlinked to corresponding entries in

http://www.guidetopharmacology.org, and are permanently archived in the Concise Guide to

PHARMACOLOGY 2019/20 [23]. 


\section{REFERENCES}

1. Chau DL, Walker V, Pai L, Cho LM. Opiates and elderly: use and side effects. Clin Interv Aging 2008; 3: 273-8.

2. Mian P, Allegaert K, Spriet I, Tibboel D, Petrovic M. Paracetamol in Older People: Towards Evidence-Based Dosing? Drugs Aging 2018.

3. Nakai Y, Makizako H, Kiyama R, Tomioka K, Taniguchi Y, Kubozono T, Takenaka T, Ohishi M. Association between Chronic Pain and Physical Frailty in Community-Dwelling Older Adults. Int J Environ Res Public Health 2019; 16.

4. Denny DL, Guido GW. Undertreatment of pain in older adults: an application of beneficence. Nurs Ethics 2012; 19: 800-9.

5. Barkin RL, Barkin SJ, Barkin DS. Pharmacotherapeutic management of pain with a focus directed at the geriatric patient. Rheum Dis Clin North Am 2007; 33: 1-31.

6. Vargas-Schaffer $\mathrm{G}$. Is the WHO analgesic ladder still valid? Twenty-four years of experience. Can Fam Physician 2010; 56: 514-7, e202-5.

7. O'Mahony D, O'Sullivan D, Byrne S, O'Connor MN, Ryan C, Gallagher P. STOPP/START criteria for potentially inappropriate prescribing in older people: version 2. Age Ageing 2015; 44: 213-8.

8. Gibb IA, Anderson BJ. Paracetamol (acetaminophen) pharmacodynamics: interpreting the plasma concentration. Arch Dis Child 2008; 93: 241-7.

9. Mian P, van Esdonk MJ, Olkkola KT, de Winter BCM, Liukas A, Spriet I, Tibboel D, Petrovic M, Koch BCP, Allegaert K. Population pharmacokinetic modelling of intravenous paracetamol in fit older people displays extensive unexplained variability. Br J Clin Pharmacol 2019; 85: 126-35.

10. Industry-Bioanalytical FaDAGf, http://www.fda. Aa, October gdDGupA, 29. In. 11. Folstein MF, Folstein SE, McHugh PR. "Mini-mental state". A practical method for grading the cognitive state of patients for the clinician. J Psychiatr Res 1975; 12: 189-98. 12. DB R, SR M, A T, RT T. Development and validation of a new Instrument

for frailty. In: Clin invest med, 2000.

13. Ellmers S.E PLRC, Notarianni L.J, Jones W. Excretion of paracetamol in fit and frail elderly people. In: J Am Geriatr Soc., 1991: 596-7.

14. Triggs EJ, Nation RL, Long A, Ashley JJ. Pharmacokinetics in the elderly. Eur J Clin Pharmacol 1975; 8: 55-62.

15. Liukas A, Kuusniemi K, Aantaa R, Virolainen P, Niemi M, Neuvonen PJ, Olkkola KT. Pharmacokinetics of intravenous paracetamol in elderly patients. Clin Pharmacokinet 2011; 50: 121-9.

16. Miners JO, Penhall R, Robson RA, Birkett DJ. Comparison of paracetamol metabolism in young adult and elderly males. Eur J Clin Pharmacol 1988; 35: 157-60.

17. Bannwarth B, Pehourcq F, Lagrange F, Matoga M, Maury S, Palisson M, Le Bars M.

Single and multiple dose pharmacokinetics of acetaminophen (paracetamol) in polymedicated very old patients with rheumatic pain. J Rheumatol 2001; 28: 182-4.

18. Heading RC, Nimmo J, Prescott LF, Tothill P. The dependence of paracetamol absorption on the rate of gastric emptying. Br J Pharmacol 1973; 47: 415-21.

19. Sabaté M, Ibáñez L, Pérez E, Vidal X, Buti M, Xiol X, Mas A, Guarner C, Forné M, Solà R, Castellote J, Rigau J, Laporte JR. Paracetamol in therapeutic dosages and acute liver injury: causality assessment in a prospective case series. BMC Gastroenterol 2011; 11: 80. 
20. Rasmussen S. NSAIDs are superior to paracetamol for osteoarthritic pain and function in a network meta-analysis. BMJ Evid Based Med 2018; 23: 40-41.

21. Prescott LF. Kinetics and metabolism of paracetamol and phenacetin. Br J Clin Pharmacol 1980; 10 Suppl 2: 291S-98S.

22. Ging P, Mikulich O, O'Reilly KM. Unexpected paracetamol (acetaminophen) hepatotoxicity at standard dosage in two older patients: time to rethink $1 \mathrm{~g}$ four times daily? Age Ageing 2016; 45: 566-7.

23. Alexander SPH, Fabbro D, Kelly E, Mathie A, Peters JA, Veale EL, Armstrong JF, Faccenda E, Harding SD, Pawson AJ, Sharman JL, Southan C, Davies JA, Collaborators C. THE CONCISE GUIDE TO PHARMACOLOGY 2019/20: Enzymes. Br J Pharmacol 2019; 176 Suppl 1: S297-S396.
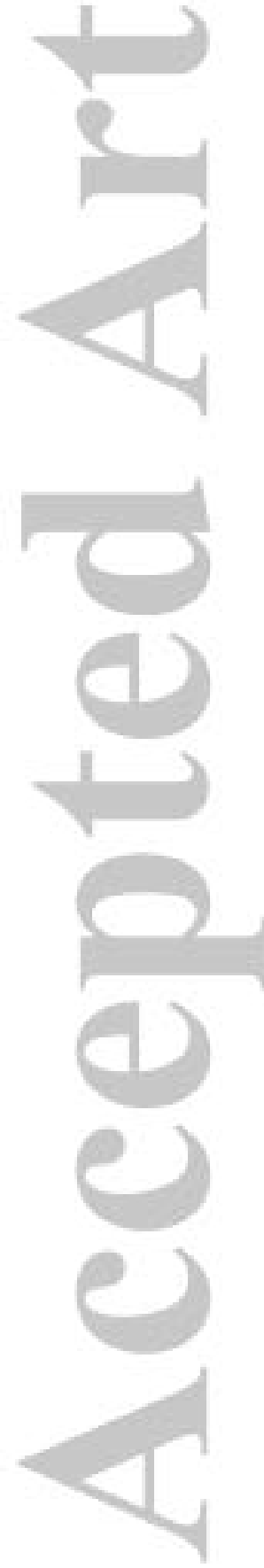
Table 1: Characteristics of included patients

\begin{tabular}{|l|l|}
\hline \multicolumn{2}{|l|}{ Table 1: Characteristics of included patients } \\
\hline Age & $86.78( \pm 4.20)$ years \\
\hline Formulation: tablet/granulate & $26 / 10(72 \% / 28 \%)$ \\
\hline Reason for paracetamol administration & \\
Pain & $32(89 \%)$ \\
Fever & $3(8 \%)$ \\
Combination pain and fever & $1(3 \%)$ \\
\hline Male/female & $16 / 20(44 \% / 56 \%)$ \\
\hline Body weight & $68.67( \pm 13.14) \mathrm{kg}$ \\
\hline Height & $1.62( \pm 0.09) \mathrm{m}$ \\
\hline BMI & $26.04( \pm 4.33)$ \\
\hline Bilirubin (total) & $0.52[0.33-0.69] \mathrm{mg} / \mathrm{dl}$ \\
\hline Bilirubin (direct) & $0.24[0.18-0.36] \mathrm{mg} / \mathrm{dl}$ \\
\hline AST & $19.5[17.00-23.75] \mathrm{U} / \mathrm{L}$ \\
\hline ALT & $14[11.00-21.00] \mathrm{U} / \mathrm{L}$ \\
\hline Gamma GT & $31[16.25-54.75] \mathrm{U} / \mathrm{L}$ \\
\hline Alkaline phosphatase & $69.50[61.75-91.00] \mathrm{U} / \mathrm{L}$ \\
\hline eGFR (CKD-EPI) & $60.42( \pm 18.31) \mathrm{ml} / \mathrm{min} / 1.73 \mathrm{~m}^{2}$ \\
\hline CrClcG & $49.21( \pm 16.46) \mathrm{ml} / \mathrm{min}$ \\
\hline Number of drugs ${ }^{\mathrm{a}}$ & $8.50[6.00-11.75]$ \\
\hline Other analgesic drugs & $17(49 \%)$ \\
NSAIDs & $1(6 \%)$ \\
Mild opioids & $9(53 \%)$ \\
Strong opioids & $7(41 \%)$ \\
\hline MMSE (/30) & $21.79( \pm 4.80)(\mathrm{n}=28)$ \\
\hline Albumin & $37.25( \pm 4.73) \mathrm{g} / \mathrm{L}$ \\
\hline Frailty (EFS) & $(\mathrm{n}=35)$ \\
O(not frail) & $1(3 \%)$ \\
1(slightly) & $4(12 \%)$ \\
2(moderate) & $12(34 \%)$ \\
3(severe) & $18(51 \%)$ \\
\hline Comorbidities & $(\mathrm{n}=36)$ \\
Diabetes mellitus & $16(44 \%)$ \\
Chronic obstructive pulmonary disease & $12(33 \%)$ \\
Heart failure & $16(44 \%)$ \\
Atrial fibrillation & $22(61 \%)$ \\
Chronic kidney disease & $18(50 \%)$ \\
Dementia & $17(47 \%)$ \\
Chronic pain & $12(33 \%)$ \\
Liver disease & $24(67 \%)$ \\
Anorexia & $9(25 \%)$ \\
Parkinson disease & $2(6 \%)$ \\
\hline NRS score & \\
t0 & $4[0-6]$ \\
t1 & $2[0-5]$ \\
t2 & $2[0-5]$ \\
t3 & $2[0-5]$ \\
t4 & $2[0-5]$ \\
\hline & \\
\hline
\end{tabular}




\begin{tabular}{|l|l}
\hline 5 & $2[0-5]$ \\
t6 & $3[0-4.5]$ \\
\hline
\end{tabular}

Data was reported as mean ( \pm SD) or median [Q1-Q3] unless stated otherwise aNumber of administrations not taken into account

BMI: Body Mass Index, AST: aspartate aminotransferase, ALT: alanine aminotransferase, Gamma GT: Gamma-glutamyltransferase, eGFR (CKD-EPI): estimated glomerular filtration rate according to chronic kidney diseaseepidemiology collaboration, CrClcG: Creatinine clearance according to Cockcroft and Gault, MMSE: Mini Mental State Examination, EFS: Edmonton Frail Scale, SD: Standard Deviation, [Q1-Q3]: Interquartile range, $\mathrm{n}$ : number of patients NRS: numeric rating scale, t0: before intake paracetamol, $\mathrm{t} 1: 30 \mathrm{~min}$ after intake, $\mathrm{t} 2: 60 \mathrm{~min}$ after intake, t3 120min after intake, t4: 240min after intake, t5: 300min after intake, t6: $360 \mathrm{~min}$ after intake of paracetamol. 
Table 2: Pharmacokinetic parameters of paracetamol compared to literature data

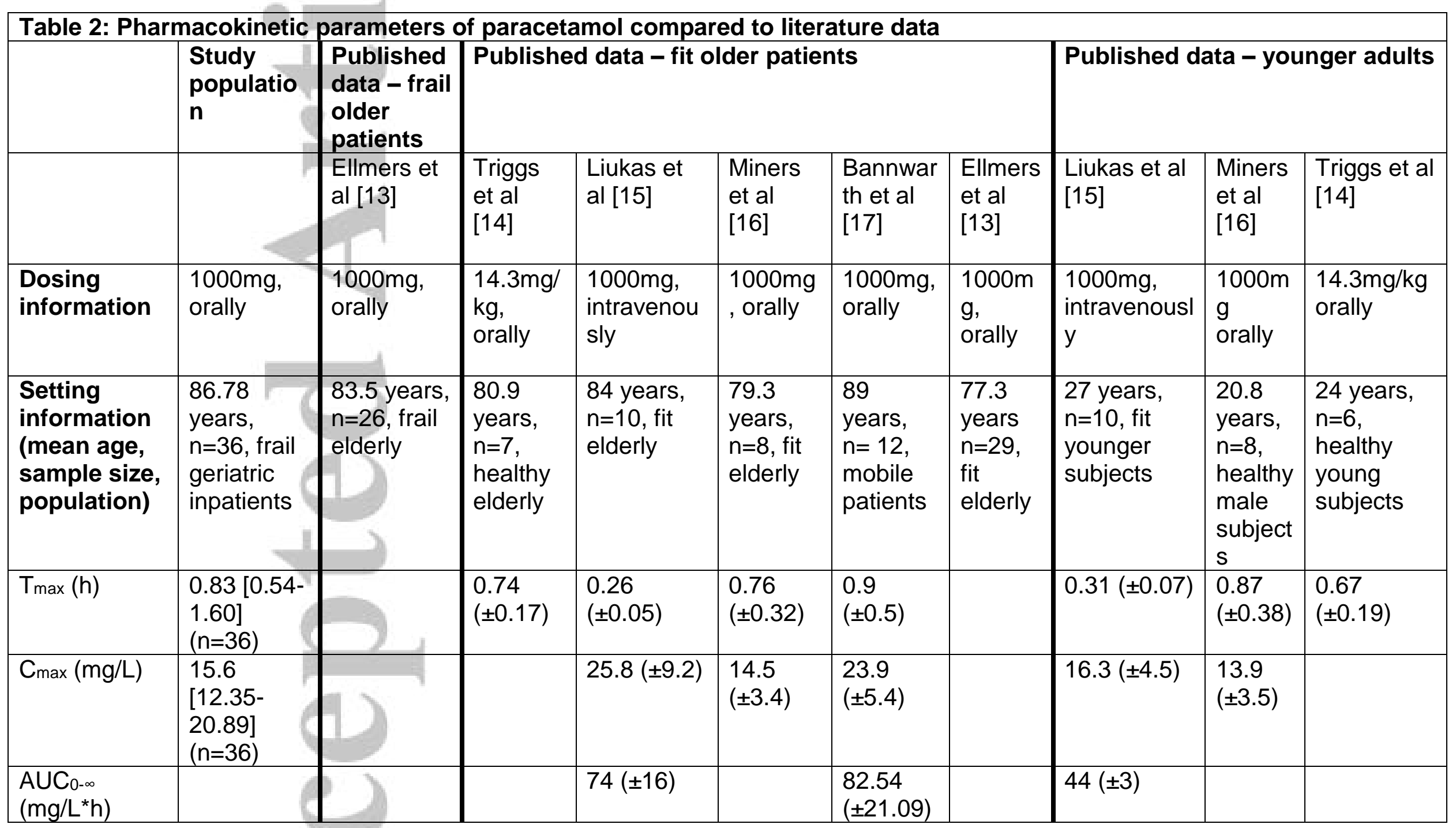




\begin{tabular}{|c|c|c|c|c|c|c|c|c|c|c|}
\hline $\begin{array}{l}\mathrm{AUC}_{0-\mathrm{t}} \\
\left(\mathrm{mg} / \mathrm{L}^{*} \mathrm{~h}\right)\end{array}$ & $\begin{array}{l}47.97 \\
{[36.99-} \\
56.06] \\
(n=33)\end{array}$ & $\begin{array}{l}74.62 \\
{[95 \% \mathrm{Cl}:} \\
42.39- \\
311.72]^{a}\end{array}$ & & & $\begin{array}{l}38.48 \\
{[95 \% \mathrm{Cl}:} \\
30.16- \\
53.15]^{\mathrm{a}}\end{array}$ & & $\begin{array}{l}56.50 \\
{[95 \% \mathrm{Cl}} \\
: 32.43- \\
219.01] \\
\mathrm{a}\end{array}$ & & $\begin{array}{l}36.48 \\
{[95 \% C} \\
\text { l: } \\
27.02- \\
56.15]^{a}\end{array}$ & \\
\hline $\begin{array}{l}\mathrm{CL} / \mathrm{F} \\
\text { (D/AUC/kg) } \\
\mathrm{ml} / \mathrm{min} / \mathrm{kg}\end{array}$ & $\begin{array}{l}5.19[4.46- \\
6.20] \\
(n=33)\end{array}$ & & & $3.3( \pm 1.0)$ & $\begin{array}{l}5.61 \\
( \pm 0.79)\end{array}$ & $\begin{array}{l}3.68 \\
( \pm 0.85)\end{array}$ & & $4.6( \pm 1.1)$ & $\begin{array}{l}6.1 \\
( \pm 1.09)\end{array}$ & \\
\hline $\begin{array}{l}\mathrm{CL} / \mathrm{F} \\
(\mathrm{ml} / \mathrm{min} / 1.73 \mathrm{~s} \\
\mathrm{qm})\end{array}$ & $\begin{array}{l}349.11 \\
{[299.79-} \\
425.62] \\
(n=33)\end{array}$ & & $\begin{array}{l}379 \\
( \pm 35)\end{array}$ & & & & & & & $477( \pm 36)$ \\
\hline Vd (L/kg) & $\begin{array}{l}0.95[0.75- \\
1.40] \\
(n=22)\end{array}$ & & $\begin{array}{l}1.05 \\
\pm 0.08\end{array}$ & $\begin{array}{l}0.92 \\
( \pm 0.26)\end{array}$ & & & & $1.04( \pm 0.22)$ & & $\begin{array}{l}1.03 \\
( \pm 0.08)\end{array}$ \\
\hline
\end{tabular}


Table 3: Pharmacokinetic parameters according to formulation

\begin{tabular}{|c|c|c|c|c|}
\hline & Total & Tablet & Granulate & $\begin{array}{l}\text { P-value (tablet } \\
\text { vs granulate) }\end{array}$ \\
\hline $\begin{array}{l}\text { Tmax }(\min ) \\
\text { median }[I Q R]\end{array}$ & $\begin{array}{l}49.5[32.25- \\
95.75] \\
(n=36)\end{array}$ & $\begin{array}{l}50.5[31.50- \\
92.50] \\
(n=26)\end{array}$ & $\begin{array}{l}42.50[33.75- \\
106.75](n=10)\end{array}$ & $P=1.000$ \\
\hline $\begin{array}{l}\mathrm{C}_{\max }(\mathrm{mg} / \mathrm{L}) \\
\text { median }[\mathrm{IQR}]\end{array}$ & $\begin{array}{l}15.6[12.35- \\
20.89] \\
(n=36)\end{array}$ & $\begin{array}{l}15.95[12.38- \\
21.19](n=26)\end{array}$ & $\begin{array}{l}15.59[10.80- \\
21.77](n=10)\end{array}$ & $P=0.698$ \\
\hline $\begin{array}{l}\mathrm{AUC}_{0-6} \\
\left(\mathrm{mg} / \mathrm{L}^{*} \mathrm{~h}\right) \\
\text { median }[\mathrm{IQR}]\end{array}$ & $\begin{array}{l}47.97[36.99- \\
56.06](n=33)\end{array}$ & $\begin{array}{l}46.56[37.83- \\
54.50](n=24)\end{array}$ & $\begin{array}{l}55.64[29.65- \\
66.16](n=9)\end{array}$ & $P=0.571$ \\
\hline \multicolumn{5}{|c|}{$\begin{array}{l}\text { Tmax: Time to reach peak concentration, } C_{\text {max: }} \text { Peak concentration, AUC: Area Under } \\
\text { the plasma concentration-time Curve, Cavg: average concentration, IQR: } \\
\text { Interquartile range }\end{array}$} \\
\hline
\end{tabular}


Table 4: Pharmacokinetic parameters of paracetamol and its metabolitesTable 4: Pharmacokinetic parameters of paracetamol and its metabolites

\begin{tabular}{|l|l|l|l|l|l|l|l|}
\hline \multicolumn{6}{|l|}{ Table 4: Pharmacokinetic parameters of paracetamol and its metabolitesTable 4: } \\
Pharmacokinetic parameters of paracetamol and its metabolites
\end{tabular}


Table 5: Determinants associated with exposure of paracetamol and its major metabolites

Table 5: Determinants associated with exposure of paracetamol and its major metabolites

\begin{tabular}{lccccc}
\hline $\begin{array}{l}\text { Regression } \\
\text { coefficient }\end{array}$ & Cavg PCT & $\begin{array}{c}\text { Cavg PCT- } \\
\text { CYS }\end{array}$ & $\begin{array}{c}\text { Cavg } \\
\text { PCT- } \\
\text { GLUC }\end{array}$ & $\begin{array}{c}\text { Cavg PCT- } \\
\text { MERC }\end{array}$ & $\begin{array}{c}\text { Cavg PCT- } \\
\text { SULF }\end{array}$ \\
\hline $\begin{array}{l}\text { Form, } \\
\text { granulate }\end{array}$ & -0.62 & 0.09 & 1.41 & 0.03 & 1.46 \\
\hline $\begin{array}{l}\text { Sex, } \\
\text { female }\end{array}$ & $1.92^{*}$ & $\mathrm{NA}$ & $\mathrm{NA}$ & $\mathrm{NA}$ & $\mathrm{NA}$ \\
\hline $\begin{array}{l}\text { Bilirubin } \\
\text { total } \\
\text { (mg/d) }\end{array}$ & $3.26^{*}$ & $\mathrm{NA}$ & $\mathrm{NA}$ & $\mathrm{NA}$ & $\mathrm{NA}$ \\
\hline $\begin{array}{l}\text { CrClcG } \\
(\boldsymbol{m I} / \mathbf{m i n})\end{array}$ & $\mathrm{NA}$ & $-0.02^{*}$ & $-0.95^{*}$ & $-0.006^{* *}$ & $-0.25^{* * *}$ \\
\hline GGT $(\mathbf{U} / \mathbf{L})$ & $\mathrm{NA}$ & $0.01^{*}$ & 0.39 & 0.002 & 0.05 \\
\hline
\end{tabular}

Cavg: average plasma concentration, PCT: paracetamol, PCT-CYS: paracetamolcysteine, PCT-GLUC: paracetamol-glucuronide, PCT-MERC: paracetamolmercaptopuric acid, PCT-SULF: paracetamol-sulphate, NA: not applicable, CrclcG: creatinine clearance according to Cockcroft and Gault GGT: gammaglutamyltransferase

${ }^{*}$ statistically significant $\left(p\right.$-value $\left.{ }^{*}<0.05 ;{ }^{* *}<0.01 ;{ }^{* * *}<0.001\right)$ 


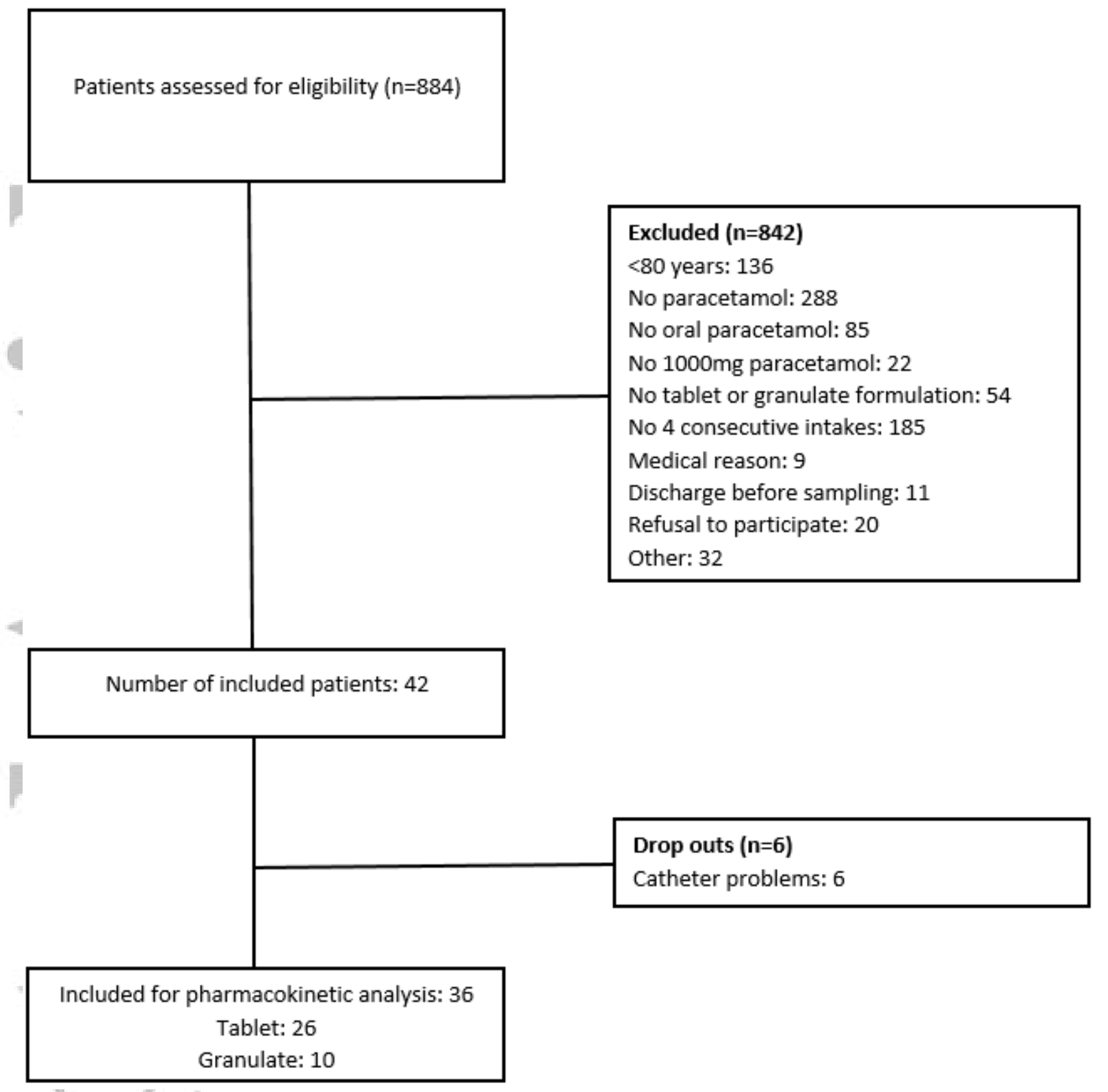

Figure 1: Patient selection process 


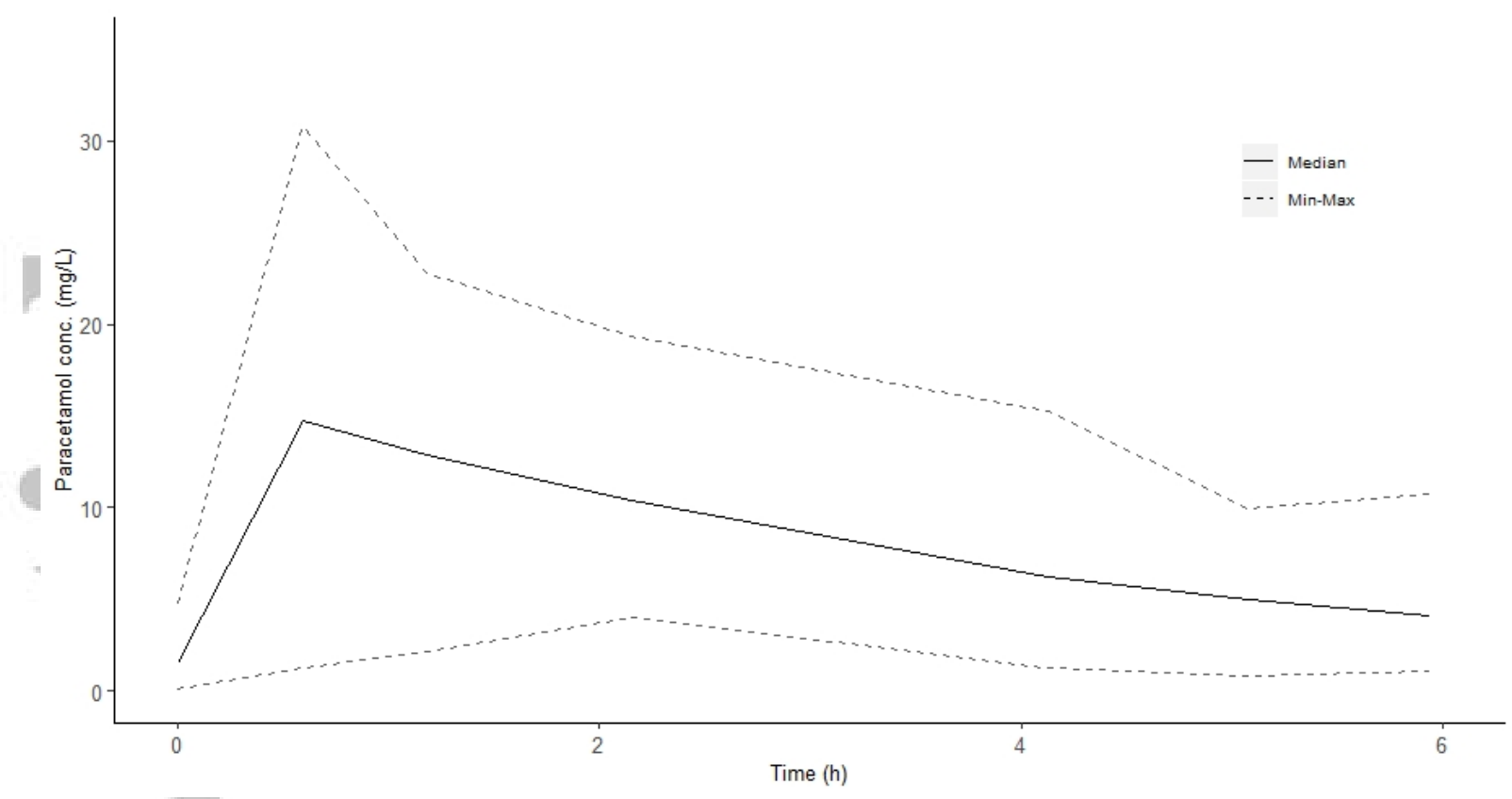

Figure 2: Median paracetamol plasma concentration-time curve. Dosing regimen: $1000 \mathrm{mg}$ TID in tablet or granulate formulation. Population: patients aged 80 years or older. Min-Max: the minimum and maximum determined concentration at each time point. 